\title{
Reviewer Acknowledgements for Global Journal of Health Science, Vol. 10, No. 6
}

Global Journal of Health Science wishes to acknowledge the following individuals for their assistance with peer review of manuscripts for this issue. Their help and contributions in maintaining the quality of the journal are greatly appreciated.

Global Journal of Health Science is recruiting reviewers for the journal. If you are interested in becoming a reviewer, we welcome you to join us. Please find the application form and details at http://recruitment.ccsenet.org and e-mail the completed application form to gjhs@ccsenet.org.

\section{Reviewers for Volume 10, Number 6}

Ama Pokuaa Fenny, University of Ghana, Ghana

Abdulbari Bener, Istaanbul University, Turkey

Angel Alfonso Velarde Lopez, University of Pennsylvania, Guatemala

António Calha, Polytechnic Institute of Portalegre, Portugal

David John Lindsay, James Cook University, Australia

Dilek Pirim, Uludag University, Turkey

Domitila Augusta Huber, Federal University of Santa Catarina, Brazil

Emad Adel Shdaifat, Imam Abdulrahman Bin Faisal University, Saudi Arabia

Evanthia Sakellari, Technological Educational Institute of Athens, Greece

Clauden Louis, University of Rochester Medical Center, United States

Georgann Valerie Weissman, Capella University, United States

Gabriel Gulis, University of Southern Denmark, Denmark

Hadii M Mamudu, East Tennessee State University, United States

Hilal Hamood Alrahbi, Diwan of Royal Court-Oman, Oman

Horng-Jyh Tsai, Kuang Tien General Hospital, Shalu Taichung, Taiwan

Hülya Yardimci, Ankara University, Turkey

Jaime Hinzpeter, Clinical Hospital University of Chile, Chile

Loray Daws, British Columbia Masterson Institute, Canada

Myo Nyein Aung, Juntendo University, Japan

Natasha Azzopardi Muscat, University of Malta, Maastricht University, Malta

Pedram Iranmanesh, Independent Researcher, Iran, Islamic Republic of

Pi-Ming Yeh, Missouri Western State University, United States

Robert Sloan, Kagoshima University Graduate School of Medical and Dental Sciences, Japan

Roger Ho, National University of Singapore, Singapore

Soon Soo Hoo, Royal North Shore Hospital, Sydney, Australia

Sara Melo, Queen's University Belfast, United Kingdom

Tan Ching Siang, Universiti Sciences Malaysia, Malaysia

Trisha Dunning, Deakin University and Barwon Health, Australia 